\title{
Solving Linear Programming Problems by Reducing to the Form with an Obvious Answer
}

\author{
G. D. Stepanov ${ }^{1}$
}

DOI: $10.18255 / 1818-1015-2021-4-434-451$

${ }^{1}$ Voronezh State Pedagogical University, 86 Lenin str, Voronezh 394043, Russia.

MSC2020: 90C05

Research article

Full text in Russian
Received July 11, 2021

After revision December 1, 2021

Accepted December 8, 2021

The article considers a method for solving a linear programming problem (LPP), which requires finding the minimum or maximum of a linear functional on a set of non-negative solutions of a system of linear algebraic equations with the same unknowns. The method is obtained by improving the classical simplex method, which when involving geometric considerations, in fact, generalizes the Gauss complete exclusion method for solving systems of equations. The proposed method, as well as the method of complete exceptions, proceeds from purely algebraic considerations. It consists of converting the entire LPP, including the objective function, into an equivalent problem with an obvious answer.

For the convenience of converting the target functional, the equations are written as linear functionals on the left side and zeros on the right one. From the coefficients of the mentioned functionals, a matrix is formed, which is called the LPP matrix. The zero row of the matrix is the coefficients of the target functional, $a_{00}$ is its free member. The algorithms are described and justified in terms of the transformation of this matrix. In calculations the matrix is a calculation table.

The method under consideration by analogy with the simplex method consists of three stages. At the first stage the LPP matrix is reduced to a special 1-canonical form. With such matrices one of the basic solutions of the system is obvious, and the target functional on it is $a_{00}$, which is very convenient. At the second stage the resulting matrix is transformed into a similar matrix with non-positive elements of the zero column (except $a_{00}$ ), which entails the non-negativity of the basic solution. At the third stage the matrix is transformed into a matrix that provides non-negativity and optimality of the basic solution.

For the second stage the analog of which in the simplex method uses an artificial basis and is the most time-consuming, two variants without artificial variables are given. When describing the first of them, along the way, a very easy-to-understand and remember analogue of the famous Farkas lemma is obtained. The other option is quite simple to use, but its full justification is difficult and will be separately published.

Keywords: linear programming; Gauss method; simplex method; LPP matrix; resolving element; choice rule; Farkas lemma; systems of linear equations; non-negative solution

\section{INFORMATION ABOUT THE AUTHORS}

\author{
Gleb D. Stepanov | orcid.org/0000-0003-3237-849X. E-mail: stpnv42@mail.ru \\ correspondence author $\mathrm{PhD}$, associate professor.
}

For citation: G. D. Stepanov, "Solving Linear Programming Problems by Reducing to the Form with an Obvious Answer”, Modeling and analysis of information systems, vol. 28, no. 4, pp. 434-451, 2021. 


\section{Решение задач линейного программирования приведением к виду с очевидным ответом}

\section{Г. Д. Степанов ${ }^{1}$}

DOI: $10.18255 / 1818-1015-2021-4-434-451$

${ }^{1}$ Воронежский государственный педагогический университет, ул. Ленина, д. 86, г. Воронеж, 394043 Россия.

УДК 519.852

Научная статья

Полный текст на русском языке
Получена 11 июля 2021 г.

После доработки 1 декабря 2021 г. Принята к публикации 8 декабря 2021 г.

В статье рассматривается способ решения задачи линейного программирования (ЗЛП), которая требует найти минимум или максимум линейного функционала на множестве неотрицательных решений системы линейных алгебраических уравнений с теми же неизвестными. Способ получен при усовершенствовании классического симплекс-метода, который, привлекая геометрические соображения фактически обобщает метод полных исключений Гаусса решения систем уравнений. Предлагаемый способ, как и метод полных исключений, исходит из чисто алгебраических соображений. Он заключается в преобразовании всей ЗЛП, включая целевую функцию, в эквивалентную задачу с очевидным ответом.

Ради удобства преобразования целевого функционала, уравнения записываются как линейные функционалы в левой части и нули в правой. Из коэффициентов упомянутых функционалов составляется матрица, которая называется матрицей ЗЛП. Нулевая строка матрицы - коэффициенты целевого функционала, $a_{00}-$ его свободный член. Описание и обоснование алгоритмов ведется в терминах преобразования этой матрицы. При вычислениях матрица является расчетной таблицей.

Рассматриваемый метод, по аналогии с симплекс-методом, состоит из трех этапов. На первом этапе матрица 3ЛП приводится к специальному 1-каноническому виду. При таких матрицах одно из базисных решений системы очевидно, и на нем целевой функционал равен $a_{00}$, что очень удобно. На втором этапе полученная матрица преобразуется в аналогичную матрицу с неположительными элементами нулевого столбца (кроме $\left.a_{00}\right)$, что влечет неотрицательность базисного решения. На третьем этапе матрица преобразуется в матрицу, обеспечивающую неотрицательность и оптимальность базисного решения.

Для второго этапа, аналог которого в симплекс-методе использует искусственный базис и является наиболее трудоемким, приводятся два варианта без искусственных переменных. При описании первого из них, попутно, получен очень простой для понимания и запоминания аналог знаменитой леммы Фаркаша. Другой вариант совсем прост в применении, но его полное обоснование сложно и будет опубликовано отдельно.

Ключевые слова: линейное программирование; метод Гаусса; симплекс-метод; матрица ЗЛП; разрешающий элемент; правило выбора; лемма Фаркаша; системы линейных уравнений; неотрицательное решение

\section{ИНФОРМАЦИЯ ОБ АВТОРАХ}

Глеб Дмитриевич Степанов | orcid.org/0000-0003-3237-849X. E-mail: stpnv42@mail.ru автор для корреспонденции $\quad$ канд.физ.-мат.наук, доцент.

Для цитирования: G. D. Stepanov, "Solving Linear Programming Problems by Reducing to the Form with an Obvious Answer", Modeling and analysis of information systems, vol. 28, no. 4, pp. 434-451, 2021.

(C) Степанов Г. Д., 2021

Эта статья открытого доступа под лицензией СС BY license (https://creativecommons.org/licenses/by/4.0/). 
В статье дается метод решения задачи линейного программирования (3ЛП), основанный на преобразовании исходной задачи к эквивалентной ЗЛП, для которой ответ практически очевиден. Его можно считать усовершенствованным симплекс-методом с более простой для понимания, запоминания и реализации вычислительной схемой, которая, в частности, не использует искусственных переменных. Для понимания метода достаточно знаний материала средней школы, поскольку при изложении легко обойтись даже без таких элементарных понятий линейной алгебры, как линейная зависимость и линейная независимость, определитель, ранг матрицы, а также и без геометрической интерпретации.

Отметим, что метод был получен в 1996 году специально для студентов с недостаточной математической подготовкой, когда в форс-мажорной ситуации автору было поручено провести занятия по исследованию операций у студентов, зачисленных,в качестве эксперимента с экстернатом, сразу на третий курс Воронежского педуниверситета. (При этом автор учитывал ощущение неоправданной сложности общепринятого изложения, возникшее у него еще при первом знакомстве с предметом по монографии [1] в далеком 1962г.) Лишь спустя многие годы автор обратил внимание на то, что полученный метод, судя по всему, специалистам незнаком: регулярно публикуются учебные пособия, в которых излагается традиционный симплекс-метод с усложненной вычислительной таблицей, с трудными для понимания и запоминания условиями оптимальности, с использованием искусственных переменных, увеличивающих размеры задач, а преподаватели соответствующих дисциплин утверждают, что "проще нельзя".

Наличие слишком большого количества публикаций по линейному программированию (только в библиографии [2] около 1500 наименований) не позволяет нам быть уверенными в новизне нашего подхода, но зато нет сомнений в том, что он был бы полезен и во многих прежних, и в новых исследованиях по данной тематике, а тем более в практике преподавания. В частности, в пункте 6 дается простой для понимания и запоминания аналог леммы Фаркаша, а в пункте 7 анонсируется простой метод нахождения неотрицательного решения систем линейных алгебраических уравнений.

Иллюстрация использования рассматриваемого в статье подхода к решению ЗЛП дана в четырех примерах пункта 8.

1. В ЗЛП, для непосредственного решения которых предназначен симплекс-метод (см.[1-8]), речь идет о минимизации или максимизации линейной функции от $n$ неизвестных на множестве неотрицательных решений системы из $m(m<n)$ линейных алгебраических уравнений от тех же неизвестных.

В отличии от классических формулировок будем считать, что оптимизируемая функция может быть не только однородной линейной формой, но и неоднородным линейным функционалом, у которого допускается и неравный нулю свободный член (аналогичные целевые функционалы используются и в $[9,10])$. Указанное чисто формальное расширение задачи позволяет преобразовывать всю задачу, а не только систему уравнений, как это делается в классическом симплекс-методе. Систему уравнений будем записывать в виде, при котором в левой части уравнений стоят линейные функционалы, а в правой - нули. Конечно, такое представление, получающееся из традиционного переносом (с изменением знака) свободных членов в левую часть, несколько непривычно, но зато удобно при преобразовании оптимизируемого функционала.

Итак, рассматривается линейный функционал

$$
a_{00}+a_{01} x_{1}+a_{02} x_{2}+\ldots+a_{0 n} x_{n}
$$


и система линейных алгебраических уравнений

$$
\begin{gathered}
a_{10}+a_{11} x_{1}+a_{12} x_{2}+\ldots+a_{1 n} x_{n}=0, \\
a_{20}+a_{21} x_{1}+a_{22} x_{2}+\ldots+a_{2 n} x_{n}=0, \\
\ldots \ldots \ldots \ldots \ldots \ldots \ldots \ldots \ldots \ldots \ldots \ldots \ldots \ldots \ldots \ldots \ldots \ldots \ldots \ldots \ldots \ldots \ldots \ldots \ldots \ldots \ldots \\
a_{m 0}+a_{m 1} x_{1}+a_{m 2} x_{2}+\ldots+a_{m n} x_{n}=0 .
\end{gathered}
$$

В ЗЛП(min)/3ЛП(max) требуется на множестве всех неотрицательных решений системы (2) найти минимум/максимум функционала (1) и какое-нибудь из неотрицательных решений, на котором этот минимум/максимум достигается. Если нужный экстремум отсутствует, то надо указать причину этого: либо система (2) несовместна, либо у системы нет неотрицательных решений, либо на непустом множестве неотрицательных решений функционал (1) не ограничен снизу (для 3ЛП(min)) или сверху (для ЗЛП $(\max ))$.

Соответствующую (1) и (2) матрицу

$$
\left(\begin{array}{ccccc}
a_{00} & a_{01} & a_{02} & \ldots & a_{0 n} \\
a_{10} & a_{11} & a_{12} & \ldots & a_{1 n} \\
a_{20} & a_{21} & a_{22} & \ldots & a_{2 n} \\
\ldots & \ldots & \ldots & \ldots & \ldots \\
a_{m 0} & a_{m 1} & a_{m 2} & \ldots & a_{m n}
\end{array}\right)
$$

будем называть матрицей ЗЛП. В наших алгоритмах такая матрица, по существу, будет расчетной таблицей.

Матрицы ЗЛП, имеющие вид

$$
\left(\begin{array}{ccccccccc}
a_{00} & a_{01} & a_{02} & \ldots & a_{0 n-m} & 0 & 0 & \ldots & 0 \\
a_{10} & a_{11} & a_{12} & \ldots & a_{1 n-m} & 1 & 0 & \ldots & 0 \\
a_{20} & a_{21} & a_{22} & \ldots & a_{2 n-m} & 0 & 1 & \ldots & 0 \\
\ldots & \ldots & \ldots & \ldots & \ldots & \ldots & \ldots & \ldots & \ldots \\
a_{m 0} & a_{m 1} & a_{m 2} & \ldots & a_{m n-m} & 0 & 0 & \ldots & 1
\end{array}\right),
$$

или приводимые к такому виду перестановкой столбцов с номерами большими нуля, будем называть матрицами канонической структуры или короче 1-каноническими. Конечно, перестановок столбцов, приводящих 1-каноническую матрицу к виду (4), может быть несколько. При выделенной каким-либо образом одной из них будем обозначать через $s_{1}, s_{2}, \ldots, s_{n}$ соответствующую перестановку исходных номеров столбцов. При этом номера $r_{1}=s_{n-m+1}, r_{2}=s_{n-m+2}, \ldots, r_{m}=s_{n}$, сами столбцы и переменные с этими номерами будем называть базисными, а номера $s_{1}, s_{2}, \ldots, s_{n-m}$, сами столбцы и переменные с этими номерами будем называть внебазисными.

Очевидно, что вектор $\left(x_{1}, x_{2}, \ldots, x_{n}\right)$, в котором все внебазисные переменные равны нулю, а базисные переменные $x_{r_{i}}=-a_{i 0}(i=1,2, \ldots m)$, является решением системы линейных ограничений. Такое решение называется базисным. Следующая основная лемма дает условия, при которых ответ для ЗЛП очевиден.

Лемма 1. Пусть матрица ЗЛП имеет каноническую структуру и X - множество неотрицательных решений системы ограничений. Тогда

$1^{0}$. На базисном решении целевой функиионал равен $a_{00}$.

$2^{0}$. Базисное решение неотрицательно тогда и только тогда, когда

$$
a_{10} \leq 0, a_{20} \leq 0, \ldots, a_{m 0} \leq 0 .
$$

$3^{0}$. Если выполнено (5) и условие

$$
a_{01} \geq 0, a_{02} \geq 0, \ldots, a_{0 n} \geq 0,
$$


то $a_{00}$ является минимумом целевого функционала на $X$.

$4^{0}$. Если выполнено (5) и условие

$$
a_{01} \leq 0, a_{02} \leq 0, \ldots, a_{0 n} \leq 0,
$$

то $a_{00}$ является максимумом цзелевого функционала на X.

$5^{0}$. Если Х непусто и существует внебазисный номер $q \neq 0$ такой, что

$$
a_{1 q} \leq 0, a_{2 q} \leq 0, \ldots, a_{m q} \leq 0,
$$

то при $a_{0 q}<0$ целевой функционал на Х неограничен снизу, а при $a_{0 q}>0$ - неограничен сверху.

Доказательство. Справедливость свойств $1^{0}, 2^{0}$ очевидна.

Для обоснования $3^{0}$ заметим, что при выполнении (6) минимум целевого функционала на множестве всех неотрицательных векторов равен $a_{00}$. Такое же значение функционал принимает на базисном решении, которое, ввиду (5), тоже неотрицательно. Вместе это означает справедливость $3^{0}$.

Свойство $4^{0}$ доказывается аналогично свойству $3^{0}$.

Для обоснования свойства $5^{0}$ обозначим через $\mathrm{y}=\left(y_{1}, y_{2}, \ldots, y_{n}\right)$ какое-нибудь неотрицательное решение системы линейных ограничений и рассмотрим зависящие от параметра $t$ вектора $z(t)=$ $\left(z_{1}(t), z_{2}(t), \ldots, z_{n}(t)\right)$ и $x(t)=y+z(t)$, где

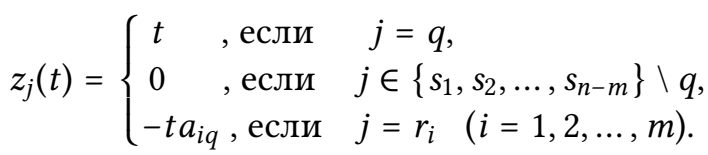

При положительных $t$ вектора $x(t)$ и $z(t)$ являются, соответственно, неотрицательными решениями системы линейных ограничений и ее однородной части. Целевой функционал на $z(t)$ равен $t a_{0 q}$ и с ростом $t$ неограниченно убывает, если $a_{0 q}<0$, или неограниченно возрастает, если $a_{0 q}>0$. Этот линейный функционал, ввиду независимости $y$ от $t$, с ростом $t$ ведет себя на $x(t)$ также, как на $z(t)$, что означает справедливость $5^{0}$. Лемма 1 доказана.

Замечание. ЗЛП $(\mathrm{min})$ и ЗЛП(max) легко сводятся друг к другу умножением целевого функционала на (-1). Тем не менее, мы даем формулировки для каждой из них. В матрицах 3ЛП, при формулировке леммы 1 и формулировках алгоритмов решения 3ЛП, прослеживается некоторая аналогия между строками и столбцами. Такая аналогия была бы еще более заметной, если в формулировку леммы добавить аналоги свойств $2^{0}-5^{0}$, относящиеся к задаче оптимизации линейного функционала на множестве неположительных решений.

2. Уточним используемое нами понятие эквивалентных ЗЛП. Две системы линейных алгебраических уравнений называются эквивалентными, если множества их решений совпадают. Две 3ЛП(min) (или две 3ЛП(max)) называются эквивалентными, если их системы уравнений эквивалентны и на каждом решении таких систем значения целевых функционалов этих задач совпадают.

Преобразовывать ЗЛП в эквивалентную задачу с очевидным ответом будем с помощью шагов исключения переменных, в основном аналогичных используемым в методе Гаусса решения систем алгебраических уравнений. Каждый шаг исключения выполняется несколькими элементарными операциями, к которым будем относить перестановку уравнений в системе ограничений, умножение уравнений системы на ненулевые множители, добавление к одним уравнениям линейных комбинаций других, вычеркивание из системы ограничений тривиальных уравнений вида $0=0$ и, наконец, добавление к целевому функционалу линейных комбинаций функционалов, стоящих в левой части уравнений системы ограничений. В терминах матрицы ЗЛП эти преобразования означают перестановку строк с номерами большими нуля, умножение строк с номерами большими нуля 
на ненулевые множители, добавление к одним строкам (включая строку с номером 0) линейной комбинации строк с номерами большими нуля.

Легко понять, что перечисленные элементарные операции преобразуют ЗЛП в эквивалентные. То, что они не меняют множество решений системы линейных ограничений задачи, очевидно. Добавление же к целевому функционалу указанной линейной комбинации может менять значение целевого функционала на каких-то векторах, но не на решениях системы линейных ограничений, поскольку на этих решениях добавляемые функционалы равны нулю.

Цель шага исключения с ведущим элементом $a_{p q} \neq 0$, где $1 \leq p \leq m$ и $1 \leq q \leq n,-$ преобразовать ЗЛП в эквивалентную ЗЛП с матрицей, у которой $a_{p q}=1$ и $a_{i q}=0$ при $i \in\{0,1, \ldots, m\} \backslash p$. (Такой шаг переводит номер q в разряд базисных, делает $\left.r_{p}=q\right)$. Для этого $p$-е уравнение умножается на $1 / a_{p q}$, затем полученное уравнение при каждом $i \in\{1, \ldots, m\} \backslash p$ умножается на $a_{i q}$ и вычитается из $i$-го уравнения, а из целевого функционала вычитается функционал, стоящий в левой части полученного $p$-го уравнения умноженный на $a_{0 q}$.

Если временно обозначить через $a_{i j}^{\mathrm{H}}$ элементы матрицы ЗЛП после шага исключения, то элементы новой матрицы выражаются через старые элементы по формулам

$$
\begin{array}{cc}
a_{p j}^{\mathrm{H}}=a_{p j} / a_{p q} & (j \in\{0,1, \ldots, n\}), \\
a_{i j}^{\mathrm{H}}=a_{i j}-a_{p j} a_{i q} / a_{p q} & (i \in\{0,1, \ldots, m\} \backslash p, \quad j \in\{0,1, \ldots, n\}) .
\end{array}
$$

Если перед шагом исключения матрица ЗЛП была 1-канонической, то и после этого шага она будет 1-канонической. При этом прежний базисный номер $r_{p}$ станет новым значением внебазисного номера $s_{q}$, а номер $q$ станет новым значением $r_{p}$, т.е. станет базисным номером.

В заключение пункта отметим, что аналоги леммы 1 и формул (9) можно было бы сформулировать и при общепринятом способе задания системы уравнений со свободными членами в правой части уравнений. В лемме пришлось бы заменить знаки неравенств (5) на противоположные, что не повлияло бы на восприятие формулировки, а в (9) для элемента $a_{00}$ уже пришлось бы использовать формулу, отличную от формулы для других элементов, что усложнило бы дальнейшее изложение.

3. Перейдем к обсуждению алгоритма решения ЗЛП. Поскольку ЗЛП и ее матрица однозначно определяют друг друга, далее обычно будем говорить о преобразованиях матрицы ЗЛП, имея в виду, что при этом преобразуется и сама ЗЛП. Лемма 1 позволяет увидеть и реализовать разные стратегии решения. Классическому симплекс-методу соответствует стратегия, состоящая из трех этапов преобразования матрицы ЗЛП. (На аналогичных этапах симплекс-метода преобразуется расширенная матрица системы уравнений и вычисляются некие дополнительные характеристики и оценки.)

Этап 1. Матрица 3 преобразуется в 1-каноническую матрицу.

Этап 2. Полученная матрица преобразуется в аналогичную матрицу, удовлетворяющую условию (5).

Этап 3. Полученная матрица, удовлетворяющая (5), преобразуется в аналогичную матрицу, дополнительно удовлетворяющую условию (6), если решается ЗЛП( $\mathrm{min})$, или (7), если решается ЗЛП(max).

Наиболее простым является этап 1 , очень похожий на основную часть метода полных исключений Гаусса решения систем уравнений. На этом этапе следует поочередно при $p=1, \ldots, m$ найти $a_{p q} \neq 0(q \geq 1)$, запомнить $r_{p}=q$ и преобразовать матрицу по формулам (9). Если при каждом $p$ ненулевой разрешающий элемент будет существовать, то система ограничений (2) совместна и матрица (3) будет преобразована в матрицу нужной структуры. Однако может случиться, что при некотором $p$ все $a_{p q}=0(q=1, \ldots, n)$. В таком случае, если $a_{p 0} \neq 0$, то система ограничений (2) несовместна, что завершает процесс поиска решения ЗЛП. Если же при некотором $p$ все $a_{p q}=0(q=0,1, \ldots, n)$, то 
p-е уравнение тривиально и его следует вычеркнуть (уменьшить на единицу номера последующих уравнений и значение $m$ ), а затем продолжить выполнение этапа 1 с того же номера $p$. Трудоемкость этапа 1 равна $O\left(n m^{2}\right)$ арифметических операций.

В симплекс-методе наиболее трудоемкий этап 2 с помощью искусственного базиса сводится к использованию этапа 3 , часто называемому основной процедурой. По этой причине мы тоже этап 3 обсудим раньше этапа 2.

К моменту начала выполнения этапа 3 матрица ЗЛП уже приведена к каноническому виду и удовлетворяет условию (5). Это означает, что базисное решение уже неотрицательно и на нем целевой функционал равен $a_{00}$. Этап представляет собой итерационный цикл, в котором поочередно по определенному правилу в матрице выбирается разрешающий элемент $a_{p q}$, а затем выполняется шаг преобразования матрицы по формулам (9). Цикл завершается, когда при выборе очередного разрешающего элемента обнаруживается (с учетом свойств $3^{0}, 4^{0}, 5^{0}$ леммы 1 ), что либо требуемый экстремум уже найден, либо отсутствует. Ясно, что наибольшего внимания в обсуждении этапа 3 заслуживает именно правило выбора разрешающего элемента.

Общее соображение, которое лежит в основе выбора разрешающего элемента, заключается в стремлении сохранить при каждом преобразовании матрицы неотрицательность базисного решения вместе с улучшением на нем значения целевого функционала, т.е. с уменьшением $a_{00}$, когда решается ЗЛП( $\mathrm{min})$, или с увеличением $a_{00}$, когда решается ЗЛП(max). Добиться такого улучшения в вырожденном случае (так называют случай, когда в (5) некоторые неравенства нестрогие) удается не всегда, а в невырожденном случае вопрос решается следующим правилом.

Правило 1. В качестве номера разрешающего столбца надо брать $q$, при котором либо $a_{0 q}<0$, если решается ЗЛП(min), либо $a_{0 q}>0$, если решается ЗЛП(max). После выбора q в качестве номера разрешающей строки надо брать такое $p$, что $a_{p q}>0 u$

$$
a_{p 0} / a_{p q} \geq a_{i 0} / a_{i q} \text { npu } a_{i q}>0(i \neq 0, p)
$$

Если выбирать $a_{p q}$ по этому правилу, то условие (10) гарантирует выполнение (5) и после шага преобразования матрицы. Значение целевого функционала на новом базисном решении будет равно $a_{00}-a_{p 0} a_{0 q} / a_{p q}$, а значит улучшится в невырожденном случае и не ухудшится в вырожденном.

Если при выборе разрешающего элемента не найдется $q$, требуемого правилом 1 (т.е. либо выполнено (6) при решении ЗЛП(min), либо (7) при решении ЗЛП(max)), то по лемме 1 искомый экстремум найден и задача решена. При уже выбранном $q$ может не существовать требуемого $p$, т.е. может выполняться условие (8). Тогда нужный экстремум отсутствует из-за неограниченности функционала и решение тоже завершено. В остальных случаях итерационный цикл продолжается. Естественно возникает вопрос о конечности этого цикла.

Здесь надо заметить, что правилом 1 значения $q$ и $p$ определяются неоднозначно. В невырожденном случае цикл завершится при любом уточнении правила 1, т.к. при каждом преобразовании матрицы значение $a_{00}$ улучшается, а значит зацикливание исключено. В вырожденном случае при неудачном уточнении этого правила зацикливание возможно. Опыт использования симплекс метода показывает, что это происходит довольно редко, но есть примеры, когда наиболее естественные уточнения правила 1 в одних вырожденных случаях приводят к успешному завершению этапа, а в других - к зацикливанию (см. пример 1 пункта 8).

4. В литературе по линейному программированию можно встретить несколько приемов, позволяющих избежать зацикливания симплекс-метода: метод возмущений или $\varepsilon$-метод (см. $[1,3,10,11])$, лексикографический метод (см. [4-6,8]), метод Бленда (см. [7, 12, 13]). Последний метод является наиболее простым, и именно его аналогом воспользуемся для исключения зацикливания. 
Правило 2. При решении ЗЛП( $\min )$ (ЗЛП( брать наименьшее $q$, при котором $a_{0 q}<0$ (> 0). После выбора $q$ в качестве номера разрешающей строки следует брать р с наименьшим $r_{p}$, из числа удовлетворяющих $a_{p q}>0 u(10)$.

Лемма 2. Пусть матрица ЗЛП имеет каноническую структуру и удовлетворяет условию (5). Тогда итерационный иикл, каждая итерация которого заключается в выборе разрешающего элемента $a_{p q}$ по правилу 2 и преобразовании матриць по формулам (9), конечен. В результате либо будет найден нужный экстремум, либо будет установлено отсутствие такого экстремума из-за неограниченности целевого функиионала.

Доказательство (от противного). Для определенности будем считать, что речь идет о ЗЛП(min). Предположим, что существуют 1-канонические матрицы, при которых имеет место зацикливание. Далее будем говорить о цикле из матриц с минимально возможными значениями $n$ и $m$. Для такого цикла каждый из $n$ столбцов и каждая из $m$ строк, номера которых больше 0 , должны периодически оказываться разрешающими, так как иначе их вычеркиванием можно уменьшить размеры матриц, не влияя на наличие цикла. В частности, у одних матриц цикла номер $n$ является базисным, а у других - нет, но зато у всех матриц $a_{10}=a_{20}=\ldots=a_{m 0}=0$.

Элементы матрицы из цикла, при преобразовании которой исходный номер $n$ переходит из разряда внебазисных в разряд базисных, будем помечать штрихом. По правилу 2 у этой матрицы $A^{\prime}$ элемент $a_{0 n}^{\prime}<0$ и $a_{0 j}^{\prime} \geq 0$ при $j \in\{1,2, \ldots, n-1\}$.

Для матрицы из цикла, при преобразовании которой исходный номер $n$ переходит из разряда базисных в разряд внебазисных, сохраним обозначение $A$. В разрешающем столбце этой матрицы только сам элемент $a_{p q}$ может стать строго положительным, так как иначе по правилу 2 из базиса выводился бы номер $<n$. Поэтому у вектора $z=\left(z_{1}, z_{2}, \ldots z_{n}\right)$ с координатами

$$
z_{j}=\left\{\begin{array}{ccc}
1 & , \text { если } & j=q, \\
0 & , \text { если } & j \in\{1,2, \ldots, n\} \backslash q \backslash\left\{s_{1}, s_{2}, \ldots, s_{m}\right\}, \\
-a_{i q} & \text {, если } & j=s_{i}(i=1,2, \ldots, m),
\end{array}\right.
$$

элемент $z_{n}=z_{s_{p}}=-a_{p q}<0$, а остальные координаты неотрицательны.

Легко проверить, что вектор $z$ является решением системы линейных ограничений ЗЛП с матрицей $A$. Поэтому, в силу эквивалентности ЗЛП с матрицами $A$ и $A^{\prime}$, значения их целевых функционалов на $z$ должны быть равны, т.е. должно выполняться равенство

$$
a_{00}+\sum_{j=1}^{n} a_{0 j} z_{j}=a_{00}^{\prime}+\sum_{j=1}^{n} a_{0 j}^{\prime} z_{j}
$$

Однако поскольку $a_{0 j}=0$ при $j \in\left\{s_{1}, s_{2}, \ldots, s_{m}\right\}, z_{q}=1$ и $z_{j}=0$ при $j \in\{1,2, \ldots, n\} \backslash q \backslash\left\{s_{1}, s_{2}, \ldots, s_{m}\right\}$, то значение левой части равно $a_{00}+a_{0 q}<a_{00}$. Значение правой части больше $a_{00}$, т.к. $a_{0 j}^{\prime} \geq 0$ и $z_{j} \geq 0$ при $1 \leq j \leq n-1, a_{0 n}^{\prime}<0, z_{n}<0, a_{00}^{\prime}=a_{00}$. Полученное противоречие завершает доказательство конечности итерационного цикла, т.е. при решении ЗЛП $(\mathrm{min})$, через конечное число шагов обязательно выполнится либо условие (6), либо условие (8).

Случай ЗЛП $(\max )$ можно рассмотреть аналогичным образом при минимальных изменениях в знаках неравенств. В этом случае цикл завершится выполнением (7) или (8). Лемма 2 доказана.

В завершение пункта отметим, что хотя итерационный цикл с правилом 2 конечен, но на вопрос о трудоемкости этапа 3, а значит и всего нашего метода, как и всего симплекс-метода, получить столь однозначный ответ не удается. Дело в том, что число всевозможных наборов базисных номеров в общем случае равно $C_{n}^{m}$, т.е. при больших $\mathrm{n}$ и т очень велико. В [7] даются специально подобранные примеры, когда при выборе разрешающего элемента по аналогу правила 1 итерационный цикл перебирает практически все комбинации базисных номеров, и там же утверждается, что такие примеры удается построить для всех известных способов уточнения этого правила. Это 
означает, что есть ЗЛП, для которых симплекс-метод весьма неэффективен. С другой стороны, при решении практических задач симплекс-метод работает очень быстро, оказывается исключительно эффективным и нет упоминаний, что когда-нибудь на практике встретилась задача, требующая перебора такого большого числа комбинаций.

5. Перейдем к обсуждению этапа 2, который должен 1-каноническую матрицу преобразовывать в аналогичную матрицу, удовлетворяющую условию (5). Мы уже отмечали, что практически во всех известных нам работах ([1-8] и др.), где излагается симплекс-метод, этап 2 сводится к этапу 3 (или объединяется с этапом 3, что менее удобно). Для этого используется метод искусственных переменных Ордена. Исключение составляют работы [9, 10], в которых ограничения заданы неравенствами. Для таких специфичных ЗЛП добавление искусственных переменных одновременно превращает неравенства в равенства и обеспечивает неотрицательность базисного решения. К исключениям еще относится редко где упоминаемая публикация [14], в которой использован прием, предполагающий возможность зацикливания, но чаще всего приводящий к нужному результату.

Метод Ордена, адаптированный к нашим формулировкам, выглядит следующим образом. Каждое уравнение с $a_{i 0}>0$, умножается на -1 и к нему добавляется искусственная переменная (для каждого такого уравнения своя), а остальные уравнения оставляются без изменений. Кроме этой искусственной системы линейных ограничений, теперь уже удовлетворяющей условия (5), формируется искусственный целевой функционал, в виде суммы искусственных переменных, добавленных к уравнениям. Ясно, что у полученной искусственной ЗЛП минимум на неотрицательных решениях не может быть меньше нуля. Если этот минимум равен нулю, то у неотрицательных решений искусственной системы линейных ограничений, на которых он достигается, все искусственные переменные равны нулю.

Полученная искусственная ЗЛП( $\mathrm{min})$ приводится к эквивалентной задаче с 1-канонической матрицей вычитанием из ее целевого функционала функционалов модифицированных уравнений. В результате получается эквивалентная ЗЛП, имеющая матрицу канонической структуры и удовлетворяющая условию (5). После этого минимум искусственной ЗЛП отыскивается с помощью основной процедуры, т.е. процедуры этапа 3. Если найденный минимум окажется положительным, то исходная ЗЛП не имеет неотрицательных решений системы ограничений. Если же минимум равен нулю, то в полученном базисном решении все искусственные переменные равны нулю и либо сразу ни одна из них не входит в число базисных, либо дополнительными преобразованиями, не нарушающими (5), они легко исключаются из числа базисных. Например, если искусственная переменная с номером $r>n$ является базисной, то $r=s_{p}$ при некотором $p$, и $a_{p 0}=0$, поскольку искусственная переменная равна нулю. Выбрав в $p$-й строке разрешающий элемент $a_{p q} \neq 0$ с $q \leq n$ и выполнив преобразование по формулам (9), мы, не меняя в матрице нулевой столбец, исключим искусственную переменную из числа базисных, заменив ее на обычную переменную.

После исключения всех искусственных переменных следует вычеркнуть из полученной матрицы столбцы, соответствующие искусственным переменным, а нулевую строку заменить строкой коэффициентов целевого функционала исходной задачи. Выполнение этапа 2 завершается исключением из этой строки всех элементов с базисными номерами.

Заметим, что мы изложили алгоритм с искусственными переменными, в основном, чтобы не быть голословными, критикуя общепринятый метод, используемый уже в течение многих десятилетий. Основным недостатком этого метода является увеличение размеров матрицы задачи, что крайне нежелательно при больших $m$ и $n$. Выбранный нами способ представления ЗЛП позволяет увидеть, что необходимость такого увеличения отсутствует.

Специфика способа в том, что все строки матрицы, включая строку с номером 0, составлены из коэффициентов функционалов. Строка коэффициентов целевого функционала отличается от 
других лишь тем, что в ней не выбираются разрешающие элементы. Учитывая это обстоятельство, с помощью основной процедуры можно постепенно увеличивать количество строк матрицы с $a_{i 0} \leq 0$ $(i \neq 0)$, в результате чего либо выполнится условие (5), либо в ходе процесса появится строка, не имеющая элементов противоположных знаков, что означает отсутствие неотрицательных решений системы ограничений.

Опишем подробнее процесс увеличения количества строк с $a_{i 0} \leq 0$.

Если в канонической матрице все $a_{i 0}>0$, то выберем разрешающий элемент $a_{1 q}$ с $a_{1 q}<0$ (при отсутствии такого элемента система линейных ограничений не имеет неотрицательных решений) и преобразуем всю матрицу по формулам (9). После этого в матрице уже есть строки с $a_{i 0} \leq 0$ и, поскольку перестановка строк является эквивалентным преобразованием, можно считать, что $a_{i 0} \leq 0$ при $1 \leq i<k$ и $a_{i 0}>0$ при $k \leq i \leq m$. Попытаемся с помощью основной процедуры, минимизировать $a_{k 0}$ на множестве неотрицательных решений первых $k-1$ уравнений. Для этого на каждом шаге процедуры разрешающие элементы надо выбирать в строках с номерами $1, \ldots, k-1$, а номер $q$ разрешающего столбца надо выбирать по $k$-й строке. При этом, чтобы матрица оставалась 1-канонической, следует преобразовывать элементы всей матрицы.

Пусть при таком применении основной процедуры минимум будет найден, т.е. будет выполнен аналог (6). Тогда либо неравенство $a_{k 0}>0$ сохранится и у исходной ЗЛП нет неотрицательных решений системы линейных ограничений, т.к. в $k$-й строке нет элементов разных знаков, либо $k$-я строка тоже попадет в число строк с $a_{i 0} \leq 0$.

Если же основная процедура установит неограниченность $a_{k 0}$ (обнаружится $a_{k q}<0$ и $a_{i q} \leq 0$ при всех $1 \leq i \leq k-1)$, то выбрав $a_{k q}$ разрешающим элементом и сделав шаг исключения опять получим $a_{k 0} \leq 0$. Описание этапа 2 и всего алгоритма завершено.

6. В данном пункте коротко обсудим некоторые следствия изложенных алгоритмов. Эти следствия касаются систем линейных алгебраических уравнений и играют важную роль в теории двойственности.

Сначала обратим внимание, что термин базисное решение относится именно к системе уравнений. Нетрудно заметить, что к базисным относятся те и только те решения системы, при которых столбцы матрицы системы, соответствующие ненулевым компонентам решения, линейно независимы.

Алгоритм этапа 1, по существу, совпадает с основной частью метода Гаусса полных исключений. Этот алгоритм либо приводит матрицу системы линейных ограничений к виду, из которого сразу находится одно из базисных решений, либо устанавливает факт отсутствия решений системы, который проявляется тем, что некоторая линейная комбинация уравнений системы представляет собой линейное уравнение, у которого свободный член отличен от нуля, а все коэффициенты при неизвестных равны нулю.

Таким образом, из существования решения системы следует существование базисного решения, и, вообще, справедлива следующая альтернатива.

Лемма 3. Всякая система линейных уравнений либо имеет (базисное) решение, либо существует линейная комбинация уравнений системы, которая не имеет решения.

Это простое утверждение можно переформулировать еще несколькими способами. Например, можно опустить взятое нами в скобки слово "базисное"или сформулировать лемму в виде критерия.

Лемма 4. Система линейных алгебраических уравнений имеет решение в том и только том случае, когда всякая линейная комбинация уравнений тоже имеет решение.

Аналогичным образом, второй вариант реализации этапа 2 (с учетом лемм 3,4) дает конструктивное доказательство эквивалентности существования неотрицательных базисных и обычных решений, а также доказательство следующей альтернативы. 
Теорема 1. Система линейных уравнений либо имеет неотрицательное решение, либо существует линейная комбинация уравнений системы, которая не имеет неотрицательного решения.

Эту теорему тоже можно переформулировать еще несколькими способами. В форме критерия она примет следующий вид.

Теорема 2. Система линейных алгебраических уравнений имеет неотрицательное решение тогда и только тогда, когда всякая линейная комбинация ее уравнений тоже имеет неотрицательное решение.

Эти лаконичные, интуитивно понятные и удобные для запоминания теоремы на самом деле являются аналогами очень известной теоремы, которая обычно называется леммой Фаркаша (см., например, $[2,4-7,10,15,16])$. Теорема 1 является аналогом этой леммы в альтернативной форме, а теорема 2 - в форме критерия. Для сравнения с теоремой 2 , приведем дословно одну из формулировок леммы Фаркаша из [2] (стр. 138).

Пусть А и $b$ соответственно матрица и вектор-столбец. Существование неотрицательного решения $x \geq 0$ системы $A x=b$ эквивалентно томy, что $y b \geq 0$ для любого вектора-строки $y$, удовлетворяющего $y A \geq 0$.

Ясно, что здесь условие “ $y b \geq 0$ для любого вектора-строки $y$, удовлетворяющего $y A \geq 0$ ” является одним из способов выразить, что у каждой линейной комбинации уравнений системы есть неотрицательные решения. Конечно, можно было бы в этом условии знаки $\geq$ поменять на $\leq$ или заменить все условия каким-либо другим эквивалентом существования у скалярных уравнений неотрицательного решения.

Аналогичным образом теорему 1 можно было бы сравнить с теоремой 7.1 из [2], которая там называется основной теоремой о линейных неравенствах и связывается с именами Фаркаша, Минковского, Каратеодори и Вейля.

Вообще, стоит отметить, что лемма Фаркаша отличается исключительным многообразием формулировок и их доказательств. Некоторые из этих формулировок довольно сложны, ввиду используемой терминологии, обозначений и особых акцентов. Надеемся, что простые формулировки теорем 1 и 2 будут способствовать лучшему пониманию более сложных формулировок.

7. Приведенный в пункте 5 второй вариант отыскания неотрицательного базисного решения более экономно использует вычислительные ресурсы, но оказалось, что есть существенно более простой способ, полученный уже при написании данной статьи и анонсированный в [17].

Еще в 1996 году, экспериментируя с программой, которая при преобразовании 1-канонической матрицы ЗЛП позволяла выбирать разрешающие элементы $a_{p q}$ в диалоговом режиме, автор заметил, что неотрицательные базисные решения очень быстро отыскивались интуитивным подбором $a_{p q}$. Фактически процесс сводился к итерационному циклу, где сначала выбиралось $p$, при котором $a_{p 0}>0$, затем выбиралось $q$, при котором $a_{p q}<0$, а затем выполнялось преобразование матрицы по формулам (9). Цикл заканчивался, когда либо выполнялось условие (5), либо появлялась строка, в которой все $a_{p j}$ были неотрицательны и $a_{p 0}>0$, что означает отсутствие неотрицательных решений. Очевидно, что если каким-то образом уточнить это правило выбора (например, в качестве $p$ и $q$ брать наименьшие из номеров, при которых $a_{p 0}>0$ и $a_{p q}<0$ ), то цикл либо закончится по одной из указанных двух причин, либо произойдет зацикливание. Однако в ходе численных экспериментов зацикливание не встречалось, и автор даже рекомендовал студентам использовать такой эвристический прием при выполнении лабораторных работ.

Уже когда работа над данной статьей завершалась, удалось получить и обосновать очень просто формулируемое правило, которое вообще исключает зацикливание.

Правило 3. Для 1-канонической матрищы в качестве номера разрешающей строки из всех $p$, $n$ ри которых $a_{p 0}>0$, надо брать номер с наименьшим $r_{p}$. При уже выбранном р надо брать наименьшее $q$, при котором $a_{p q}<0$. 
Теорема 3. Для 1-канонических матриц итерационный цикл, тело которого состоит из выбора разрешающего элемента по правилу 3 и последующего шага исключения, конечен.

Еще раз обратим внимание, что в теореме, по сути, речь идет о конечности простого алгоритма решения важной задачи отыскания неотрицательного базисного решения, которая до сих пор во всех известных нам публикациях решалась методом искусственного базиса или еще более сложными методами. Несмотря на простоту приведенного правила выбора разрешающего элемента, доказательство теоремы весьма непросто, связано с обоснованием еще нескольких алгоритмов решения ЗЛП и требует написания отдельной статьи.

8. В этом пункте приведем четыре примера, иллюстрирующих решение ЗЛП с использованием рассмотренного в статье подхода. Напомним, что алгоритм заключается в приведении ЗЛП к виду, при котором выполнены условия леммы 1, и состоит из трех этапов, некоторые из которых могут не понадобиться. Каждый этап представляет из себя цикл, в теле которого соответствующим образом выбирается разрешающий элемент, а затем матрица преобразуется по формулам (9). Нумерация строк и столбцов начинается с нуля. Разрешающий элемент берется из строк и столбцов с номерами больше нуля. Значение целевого функционала на текущем базисном решении присутствует в таблице в явном виде как элемент $a_{00}$. Мы будем на каждой итерации помечать разрешающий столбец звездочкой в нулевой строке, разрешающую строку - звездочкой в нулевом столбце, а сам разрешающий элемент - двумя звездочками.

\section{Пример 1.}

Требуется решить ЗЛП $(\min )$ и ЗЛП $(\max )$ с 1-канонической матрицей

$$
\left(\begin{array}{rrrrrrr}
1 & 0 & 0 & 5 & 12 & 18 & -41 \\
0 & 0 & 1 & -3 & -5 & 7 & 16 \\
0 & 1 & 0 & 1 & 2 & -3 & 7
\end{array}\right) .
$$

Эти задачи вырожденные, условие (5) выполняется автоматически и требуется выполнять лишь этап 3. Поскольку при исключении переменных свободный член целевого функционала не меняется, то максимум и минимум могут иметь лишь значение $a_{00}=1$, хотя могут и отсутствовать.

Сначала решим ЗЛП(min), выбирая в качестве $p$ и $q$ наименьшие номера, удовлетворяющие правилу 1.

\begin{tabular}{|r|r|r|r|r|r|r|}
\hline 1 & 0 & 0 & 5 & 12 & $-18^{*}$ & -41 \\
\hline $0^{*}$ & 0 & 1 & -3 & -5 & $7^{* *}$ & 16 \\
\hline 0 & 1 & 0 & 1 & 2 & -3 & -7 \\
\hline 1 & 0 & $18 / 7$ & $-19 / 7^{*}$ & $-6 / 7$ & 0 & $1 / 7$ \\
\hline 0 & 0 & $1 / 7$ & $-3 / 7$ & $-5 / 7$ & 1 & $16 / 7$ \\
\hline 0 & 1 & $3 / 7$ & $-2 / 7$ & $-1 / 7$ & 0 & $-1 / 7$ \\
\hline
\end{tabular}

Уже на второй итерации, после выбора $q=3$ в столбце с этим номером нет положительных элементов. Это означает, что выполнено условие (8) и целевая функция неограничена снизу.

Применяя аналогичный способ уточнения правила 1 для решения 3ЛП(max), получаем следующую последовательность итераций. 


\begin{tabular}{|r|r|r|r|r|r|r|}
\hline 1 & 0 & 0 & $5^{*}$ & 12 & -18 & -41 \\
\hline 0 & 0 & 1 & -3 & -5 & 7 & 16 \\
\hline $0^{*}$ & 1 & 0 & $1^{* *}$ & 2 & -3 & -7 \\
\hline 1 & -5 & 0 & 0 & $2^{*}$ & -3 & -6 \\
\hline $0^{*}$ & 3 & 1 & 0 & $1^{* *}$ & -2 & -5 \\
\hline 0 & 1 & 0 & 1 & 2 & -3 & -7 \\
\hline 1 & -11 & -2 & 0 & 0 & $1^{*}$ & 4 \\
\hline 0 & 3 & 1 & 0 & 1 & -2 & -5 \\
\hline $0^{*}$ & -5 & -2 & 1 & 0 & $1^{* *}$ & 3 \\
\hline 1 & -6 & 0 & -1 & 0 & 0 & $1^{*}$ \\
\hline $0^{*}$ & -7 & -3 & 2 & 1 & 0 & $1^{* *}$ \\
\hline 0 & -5 & -2 & 1 & 0 & 1 & 3 \\
\hline \hline 1 & $1^{*}$ & 3 & -3 & -1 & 0 & 0 \\
\hline 0 & -7 & -3 & 2 & 1 & 0 & 1 \\
\hline $0^{*}$ & $16^{* *}$ & 7 & -5 & -3 & 1 & 0 \\
\hline 1 & 0 & $41 / 16^{*}$ & $-43 / 16$ & $-13 / 16$ & $-1 / 16$ & 0 \\
\hline $0^{*}$ & 0 & $1 / 16^{* *}$ & $-3 / 16$ & $-5 / 16$ & $7 / 16$ & 1 \\
\hline 0 & 1 & $7 / 16$ & $-5 / 16$ & $-3 / 16$ & $1 / 16$ & 0 \\
\hline 1 & 0 & 0 & 5 & 12 & -18 & -41 \\
\hline 0 & 0 & 1 & -3 & -5 & 7 & 16 \\
\hline 0 & 1 & 0 & 1 & 2 & -3 & -7 \\
\hline
\end{tabular}

После шестой итерации мы получили исходную матрицу, т.е. произошло зацикливание. Чтобы этого не случилось, надо было использовать правило 2, которое в любом случае исключает зацикливание. Применяя это правило к нашей задаче, убеждаемся, что вплоть до шестой итерации выбираются те же разрешающие элементы. На шестой итерации, после выбора $q=2$, по правилу 2 надо выбрать $p=2$, т.к. $r_{1}=6$, а $r_{2}=1$. Таким образом, начиная с шестой итерации, вычисления будут иметь следующий вид.

\begin{tabular}{|r|r|r|r|r|r|r|}
\hline 1 & 0 & $41 / 16^{*}$ & $-43 / 16$ & $-13 / 16$ & $-1 / 16$ & 0 \\
\hline 0 & 0 & $1 / 16$ & $-3 / 16$ & $-5 / 16$ & $7 / 16$ & 1 \\
\hline $0^{*}$ & 1 & $7 / 16^{* *}$ & $-5 / 16$ & $-3 / 16$ & $1 / 16$ & 0 \\
\hline 1 & $-41 / 7$ & 0 & $-6 / 7$ & $2 / 7^{*}$ & $-3 / 7$ & 0 \\
\hline 0 & $-1 / 7$ & 0 & $-1 / 7$ & $-2 / 7$ & $3 / 7$ & 1 \\
\hline 0 & $16 / 7$ & 1 & $-5 / 7$ & $-3 / 7$ & $1 / 7$ & 0 \\
\hline
\end{tabular}

На седьмой итерации выбирается $q=4$, а разрешающего элемента выбрать не удается, т. к. в четвертом столбце вне нулевой строки нет положительных элементов. Это означает, что выполнено условие (8) для ЗЛП( $\max )$ и целевая функция сверху тоже неограничена. Конец примера.

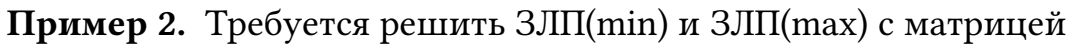

$$
\left(\begin{array}{rrrrrrrr}
0 & -2 & -2 & 1 & -2 & -1 & 0 & -1 \\
1 & -2 & -1 & -1 & -2 & -1 & -2 & 1 \\
2 & -2 & -1 & 1 & -1 & 1 & -1 & 0 \\
3 & 1 & 0 & -1 & -2 & -1 & -1 & -2
\end{array}\right) .
$$

Этап 1 совпадает с основной частью метода полных исключений Гаусса. 


\begin{tabular}{|r|r|r|r|r|r|r|r|}
\hline 0 & $-2^{*}$ & -2 & 1 & -2 & -1 & 0 & -1 \\
\hline $1^{*}$ & $-2^{* *}$ & -1 & -1 & -2 & -1 & -2 & 1 \\
\hline 2 & -2 & -1 & 1 & -1 & 1 & -1 & 0 \\
\hline 3 & 1 & 0 & -1 & -2 & -1 & -1 & -2 \\
\hline-1 & 0 & -1 & $2^{*}$ & 0 & 0 & 2 & -2 \\
\hline$-1 / 2$ & 1 & $1 / 2$ & $1 / 2$ & 1 & $1 / 2$ & 1 & $-1 / 2$ \\
\hline $1^{*}$ & 0 & 0 & $2^{* *}$ & 1 & 2 & 1 & -1 \\
\hline $7 / 2$ & 0 & $-1 / 2$ & $-3 / 2$ & -3 & $-3 / 2$ & -2 & $-3 / 2$ \\
\hline-2 & 0 & $-1^{*}$ & 0 & -1 & -2 & 1 & -1 \\
\hline$-3 / 4$ & 1 & $1 / 2$ & 0 & $3 / 4$ & 0 & $3 / 4$ & $-1 / 4$ \\
\hline $1 / 2$ & 0 & 0 & 1 & $1 / 2$ & 1 & $1 / 2$ & $-1 / 2$ \\
\hline $17 / 4^{*}$ & 0 & $-1 / 2^{* *}$ & 0 & $-9 / 4$ & 0 & $-5 / 4$ & $-9 / 4$ \\
\hline$-21 / 2$ & 0 & 0 & 0 & $7 / 2$ & -2 & $7 / 2$ & $7 / 2$ \\
\hline $7 / 2$ & 1 & 0 & 0 & $-3 / 2$ & 0 & $-1 / 2$ & $-5 / 2$ \\
\hline $1 / 2$ & 0 & 0 & 1 & $1 / 2$ & 1 & $1 / 2$ & $-1 / 2$ \\
\hline$-17 / 2$ & 0 & 1 & 0 & $9 / 2$ & 0 & $5 / 2$ & $9 / 2$ \\
\hline
\end{tabular}

Теперь матрица ЗЛП является 1-канонической. Этап 2 будем реализовывать способом, изложенном в пункте 7, т. е. итерационным циклом с правилом 3.

\begin{tabular}{|c|c|r|r|r|r|r|r|}
\hline$-21 / 2$ & 0 & 0 & 0 & $7 / 2^{*}$ & -2 & $7 / 2$ & $7 / 2$ \\
\hline $7 / 2^{*}$ & 1 & 0 & 0 & $-3 / 2^{* *}$ & 0 & $-1 / 2$ & $-5 / 2$ \\
\hline $1 / 2$ & 0 & 0 & 1 & $1 / 2$ & 1 & $1 / 2$ & $-1 / 2$ \\
\hline$-17 / 2$ & 0 & 1 & 0 & $9 / 2$ & 0 & $5 / 2$ & $9 / 2$ \\
\hline$-7 / 3$ & $7 / 3$ & 0 & 0 & 0 & -2 & $7 / 3$ & $-7 / 3^{*}$ \\
\hline$-7 / 3$ & $-2 / 3$ & 0 & 0 & 1 & 0 & $1 / 3$ & $5 / 3$ \\
\hline $5 / 3$ & $1 / 3$ & 0 & 1 & 0 & 1 & $1 / 3$ & $-4 / 3$ \\
\hline $2^{*}$ & 3 & 1 & 0 & 0 & 0 & 1 & $-3^{* *}$ \\
\hline$-35 / 9$ & $0^{*}$ & $-7 / 9$ & 0 & 0 & -2 & $14 / 9$ & 0 \\
\hline$-11 / 9$ & 1 & $5 / 9$ & 0 & 1 & 0 & $8 / 9$ & 0 \\
\hline $7 / 9^{*}$ & $-1^{* *}$ & $-4 / 9$ & 1 & 0 & 1 & $-1 / 9$ & 0 \\
\hline$-2 / 3$ & -1 & $-1 / 3$ & 0 & 0 & 0 & $-1 / 3$ & 1 \\
\hline$-35 / 9$ & 0 & $-7 / 9$ & 0 & 0 & -2 & $14 / 9$ & 0 \\
\hline$-4 / 9$ & 0 & $1 / 9$ & 1 & 1 & 1 & $7 / 9$ & 0 \\
\hline$-7 / 9$ & 1 & $4 / 9$ & -1 & 0 & -1 & $1 / 9$ & 0 \\
\hline$-13 / 9$ & 0 & $1 / 9$ & -1 & 0 & -1 & $-2 / 9$ & 1 \\
\hline
\end{tabular}

Получили 1-каноническую матрицу 3ЛП, удовлетворяющую условию (5). Этап 3 будем реализовывать итерационным циклом с правилом 2. Сначала решим 3ЛП(min). 


\begin{tabular}{|c|r|r|r|r|r|r|r|}
\hline$-35 / 9$ & 0 & $-7 / 9^{*}$ & 0 & 0 & -2 & $14 / 9$ & 0 \\
\hline$-4 / 9$ & 0 & $1 / 9$ & 1 & 1 & 1 & $7 / 9$ & 0 \\
\hline$-7 / 9^{*}$ & 1 & $4 / 9^{* *}$ & -1 & 0 & -1 & $1 / 9$ & 0 \\
\hline$-13 / 9$ & 0 & $1 / 9$ & -1 & 0 & -1 & $-2 / 9$ & 1 \\
\hline$-21 / 4$ & $7 / 4$ & 0 & $-7 / 4^{*}$ & 0 & $-15 / 4$ & $7 / 4$ & 0 \\
\hline$-1 / 4^{*}$ & $-1 / 4$ & 0 & $5 / 4^{* *}$ & 1 & $5 / 4$ & $3 / 4$ & 0 \\
\hline$-7 / 4$ & $9 / 4$ & 1 & $-9 / 4$ & 0 & $-9 / 4$ & $1 / 4$ & 0 \\
\hline$-5 / 4$ & $-1 / 4$ & 0 & $-3 / 4$ & 0 & $-3 / 4$ & $-1 / 4$ & 1 \\
\hline$-28 / 5$ & $7 / 5$ & 0 & 0 & $7 / 5$ & $-2^{*}$ & $14 / 5$ & 0 \\
\hline$-1 / 5^{*}$ & $-1 / 5$ & 0 & 1 & $4 / 5$ & $1^{* *}$ & $3 / 5$ & 0 \\
\hline$-11 / 5$ & $9 / 5$ & 1 & 0 & $9 / 5$ & 0 & $8 / 5$ & 0 \\
\hline$-7 / 5$ & $-2 / 5$ & 0 & 0 & $3 / 5$ & 0 & $1 / 5$ & 1 \\
\hline-6 & 1 & 0 & 2 & 3 & 0 & 4 & 0 \\
\hline$-1 / 5$ & $-1 / 5$ & 0 & 1 & $4 / 5$ & 1 & $3 / 5$ & 0 \\
\hline$-11 / 5$ & $9 / 5$ & 1 & 0 & $9 / 5$ & 0 & $8 / 5$ & 0 \\
\hline$-7 / 5$ & $-2 / 5$ & 0 & 0 & $3 / 5$ & 0 & $1 / 5$ & 1 \\
\hline
\end{tabular}

Условие (6) выполнено. По лемме 1 минимум целевого функционала на неотрицательных решениях равен $a_{00}=-6$. Он достигается на неотрицательном базисном решении $x=(0,11 / 5,0,0,1 / 5,0,7 / 5)$.

Решим ЗЛП(max).

\begin{tabular}{|c|r|r|r|r|r|r|r|}
\hline$-35 / 9$ & 0 & $-7 / 9$ & 0 & 0 & -2 & $14 / 9^{*}$ & 0 \\
\hline$-4 / 9^{*}$ & 0 & $1 / 9$ & 1 & 1 & 1 & $7 / 9^{* *}$ & 0 \\
\hline$-7 / 9$ & 1 & $4 / 9$ & -1 & 0 & -1 & $1 / 9$ & 0 \\
\hline$-13 / 9$ & 0 & $1 / 9$ & -1 & 0 & -1 & $-2 / 9$ & 1 \\
\hline-3 & 0 & -1 & -2 & -2 & -4 & 0 & 0 \\
\hline$-4 / 7^{*}$ & 0 & $1 / 7$ & $9 / 7$ & $9 / 7$ & $9 / 7$ & 1 & 0 \\
\hline$-5 / 7$ & 1 & $3 / 7$ & $-8 / 7$ & $-1 / 7$ & $-8 / 7$ & 0 & 0 \\
\hline$-11 / 7$ & 0 & $1 / 7$ & $-5 / 7$ & $2 / 7$ & $-5 / 7$ & 0 & 1 \\
\hline
\end{tabular}

Теперь условие (7) выполнено. Максимум целевого функционала на неотрицательных решениях равен $a_{00}=-3$. Он достигается на неотрицательном базисном решении $x=(5 / 7,0,0,0,0,4 / 7,11 / 7)$. Задачи примера 2 решены.

Пример 3. Требуется решить ЗЛП(min) с матрицей

$$
\left(\begin{array}{rrrrrrrr}
-2 & 1 & 1 & -2 & -2 & 0 & -1 & 0 \\
0 & 0 & -1 & 1 & -2 & 0 & -2 & -2 \\
1 & 1 & -2 & -1 & 1 & 0 & -2 & -1 \\
-1 & -1 & 1 & 1 & -1 & 1 & 0 & -2 \\
1 & -2 & 2 & 3 & -4 & 1 & 0 & -3
\end{array}\right) .
$$

Этап 1. Опять используем схему полных исключений Гаусса. 


\begin{tabular}{|r|r|r|r|r|r|r|r|}
\hline-2 & 1 & $1^{*}$ & -2 & -2 & 0 & -1 & 0 \\
\hline $0^{*}$ & 0 & $-1^{* *}$ & 1 & -2 & 0 & -2 & -2 \\
\hline 1 & 1 & -2 & -1 & 1 & 0 & -2 & -1 \\
\hline-1 & -1 & 1 & 1 & -1 & 1 & 0 & -2 \\
\hline 1 & -2 & 2 & 3 & -4 & 1 & 0 & -3 \\
\hline-2 & $1^{*}$ & 0 & -1 & -4 & 0 & -3 & -2 \\
\hline 0 & 0 & 1 & -1 & 2 & 0 & 2 & 2 \\
\hline $1^{*}$ & $1^{* *}$ & 0 & -3 & 5 & 0 & 2 & 3 \\
\hline-1 & -1 & 0 & 2 & -3 & 1 & -2 & -4 \\
\hline 1 & -2 & 0 & 5 & -8 & 1 & -4 & -7 \\
\hline-3 & 0 & 0 & $2^{*}$ & -9 & 0 & -5 & -5 \\
\hline 0 & 0 & 1 & -1 & 2 & 0 & 2 & 2 \\
\hline 1 & 1 & 0 & -3 & 5 & 0 & 2 & 3 \\
\hline $0^{*}$ & 0 & 0 & $-1^{* *}$ & 2 & 1 & 0 & -1 \\
\hline 3 & 0 & 0 & -1 & 2 & 1 & 0 & -1 \\
\hline-3 & 0 & 0 & 0 & -5 & 2 & -5 & -7 \\
\hline 0 & 0 & 1 & 0 & 0 & -1 & 2 & 3 \\
\hline 1 & 1 & 0 & 0 & -1 & -3 & 2 & 6 \\
\hline 0 & 0 & 0 & 1 & -2 & -1 & 0 & 1 \\
\hline $3^{*}$ & 0 & 0 & 0 & 0 & 0 & 0 & 0 \\
\hline
\end{tabular}

В последней строке элемент нулевого столбца положителен, а остальные элементы равны нулю. Это означает, что система линейных ограничений поставленной задачи не имеет решений. Конец примера 3.

Пример 4. Решить ЗЛП(min) с 1-канонической матрицей

$$
\left(\begin{array}{rrrrrrrr}
-1 / 2 & 0 & 0 & 0 & 3 / 2 & 0 & 1 / 2 & -5 / 2 \\
7 / 8 & 1 & 0 & 0 & -7 / 8 & -5 / 4 & -9 / 8 & -3 / 8 \\
-3 / 8 & 0 & 1 & 0 & 11 / 8 & 5 / 4 & 5 / 8 & -1 / 8 \\
3 / 8 & 0 & 0 & 1 & 5 / 8 & 3 / 4 & 3 / 8 & 1 / 8
\end{array}\right) .
$$

Поскольку матрица 3ЛП является 1-канонической, решение начинается сразу с этапа 2, который опять будем реализовывать итерационным циклом с правилом 3. 


\begin{tabular}{|r|r|r|r|r|r|r|r|}
\hline$-1 / 2$ & 0 & 0 & 0 & $3 / 2^{*}$ & 0 & $1 / 2$ & $-5 / 2$ \\
\hline $7 / 8^{*}$ & 1 & 0 & 0 & $-7 / 8^{* *}$ & $-5 / 4$ & $-9 / 8$ & $-3 / 8$ \\
\hline$-3 / 8$ & 0 & 1 & 0 & $11 / 8$ & $5 / 4$ & $5 / 8$ & $-1 / 8$ \\
\hline $3 / 8$ & 0 & 0 & 1 & $5 / 8$ & $3 / 4$ & $3 / 8$ & $1 / 8$ \\
\hline 1 & $12 / 7$ & 0 & 0 & 0 & $-15 / 7^{*}$ & $-10 / 7$ & $-22 / 7$ \\
\hline-1 & $-8 / 7$ & 0 & 0 & 1 & $10 / 7$ & $9 / 7$ & $3 / 7$ \\
\hline $1^{*}$ & $11 / 7$ & 1 & 0 & 0 & $-5 / 7^{* *}$ & $-8 / 7$ & $-5 / 7$ \\
\hline 1 & $5 / 7$ & 0 & 1 & 0 & $-1 / 7$ & $-3 / 7$ & $-1 / 7$ \\
\hline-2 & -3 & $-3^{*}$ & 0 & 0 & 0 & 2 & -1 \\
\hline 1 & 2 & 2 & 0 & 1 & 0 & -1 & -1 \\
\hline$-7 / 5$ & $-11 / 5$ & $-7 / 5$ & 0 & 0 & 1 & $8 / 5$ & 1 \\
\hline $4 / 5^{*}$ & $2 / 5$ & $-1 / 5^{* *}$ & 1 & 0 & 0 & $-1 / 5$ & 0 \\
\hline-14 & -9 & 0 & -15 & 0 & 0 & $5^{*}$ & -1 \\
\hline $9^{*}$ & 6 & 0 & 10 & 1 & 0 & $-3^{* *}$ & -1 \\
\hline-7 & -5 & 0 & -7 & 0 & 1 & 3 & 1 \\
\hline-4 & -2 & 1 & -5 & 0 & 0 & 1 & 0 \\
\hline 1 & 1 & 0 & $5 / 3$ & $5 / 3$ & 0 & 0 & $-8 / 3$ \\
\hline-3 & -2 & 0 & $-10 / 3$ & $-1 / 3$ & 0 & 1 & $1 / 3$ \\
\hline $2^{*}$ & 1 & 0 & 3 & 1 & 1 & 0 & 0 \\
\hline-1 & 0 & 1 & $-5 / 3$ & $1 / 3$ & 0 & 0 & $-1 / 3$ \\
\hline
\end{tabular}

В строке с номером 2 все элементы неотрицательны, а элемент нулевого столбца строго положителен. Это означает, что у системы линейных ограничений вообще нет неотрицательных решений. Конец примера 4.

В заключение отметим, что выбор разрешающего элемента на этапе 1 и на этапе 2, при использовании правила 3, не зависит от целевого функционала. Поэтому на этих этапах при ручных вычислениях, для экономии, нулевую строку можно не преобразовывать, а преобразовать ее тогда, когда уже будет выполняться условие (5), т.е. непосредственно перед этапом 3.

\section{References}

[1] S. I. Gass, Linear Programming: Methods and Applications. McGraw-Hill: New York, 1958.

[2] A. Schrijver, Theory of linear and integer programming. Moscow: Mir, 1991.

[3] D. B. Yudin and E. G. Holstein, Linear Programming: Theory and finite methods. Moscow: Fizmatlit, 1963.

[4] G. B. Dantzig, Linear Programming and Extensions. Princeton University Press, 1963.

[5] T. S. Hu, Integer programming and network flows. Addison-Wesley, 1969.

[6] S. A. Ashmanov, Linear Programming. Moscow: Nauka, 1981.

[7] C. H. Papadimitriou and K. Steiglitz, Combinatorial optimization: algorithms and complexity. Prentice-Hall, 1982.

[8] F. P. Vasiliev and A. Y. Ivanitsky, Linear Programming. MCCME, 2020.

[9] E. Stiefel, "Note on Jordan elimination, linear programming and Tchebycheff approximation", Numerische Mathematik, vol. 2, no. 1, pp. 1-17, 1960.

[10] S. I. Zukhovitsky and L. I. Avdeeva, Linear and Convex Programming. Moscow: Nauka, 1967. 
[11] A. Charnes, "Optimality and degeneracy in linear programming", Econometrica: fournal of the Econometric Society, vol. 20, no. 2, pp. 160-170, 1952.

[12] R. G. Bland, "New finite pivoting rules for the simplex method", Mathematics of Operations Research, vol. 2, no. 2, pp. 103-107, 1977.

[13] H. W. Kuhn, Class Notes. Princeton University, 1976.

[14] A. S. Barsov, What is Linear Programming. Moscow: Fizmatgiz, 1959.

[15] V. G. Karmanov, Mathematical Programming. Moscow: Fizmatlit, 2004.

[16] Y. G. Evtushenko, A. A. Tret'yakov, and E. E. Tyrtyshnikov, "New approach to Farkas' theorem of the alternative”, in Doklady Mathematics, vol. 99, Springer, 2019, pp. 208-210.

[17] G. D. Stepanov, "A Simple Algorithm for Finding a Non-negative Basic Solution of a System of Linear Algebraic Equations", Modeling and analysis of information systems, vol. 28, no. 3, pp. 234-237, 2021. 